trypomastigotes and emerge into the peripheral blood to produce the 72-96 hour parasitaemia. During the 144-168 hour remission, a second "crop" of rounded forms develops in the choroid plexus to initiate similarly the relapse at 192 hours.

Ormerod and Venkatesan suggest that this cycle occurs only in strains of subspecies of $T$. brucei which produce relatively chronic infections, such as the West African forms ( $T$. b. gambiense) and those from the southern part of the range of the East African form $(T . b$. rhodesiense); it probably does not occur in the virulent strains of $T . b$. rhodesiense which occur further north, nor in strains which have become "rodentadapted" by prolonged maintenance in laboratory hosts. This might explain the failure of most previous investigators to observe it.

Ormerod and Venkatesan's evidence for the presence of these forms in the choroid plexus seems incontrovertible. But several questions remain to be answered about the proposed life cycle. First, the viability of the "occult" amastigotes and sphaeromastigotes is suggested so far only by their staining properties and the motility of the sphaeromastigotes. Second, it is difficult to understand why the trypomastigotes inoculated into the rat to infect it do not themselves immediately commence binary fission, as they do after the first "occult" cycle. Third, the work is based on infections initiated artificially by the inoculation of infected blood; what happens naturally when mammals are infected by tsetse flies? If these stages are truly integral to the life cycle, their discovery could clearly be of great importance in relation to chemotherapy and also to the organism's evasion of its host's immune response.

\section{CHROMOSOMES}

\section{Going Macro}

from a Correspondent

UNDER certain circumstances, one of the two genetically identical micronuclei of the ciliated protozoon Stylonychia changes into a macronucleus. The DNA content increases about fourteenfold and results in the formation of polytene chromosomes which become transected into short lengths by membranous partitions between each of the chromomeres. Immediately after the breakup of the polytene chromosomes, 93 per cent of the DNA is destroyed and after a few hours the remaining 7 per cent of the macronuclear DNA replicates many times until finally the DNA content is increased to about sixty-five times the amount in the original micronucleus.

Prescott et al. (Chromosoma, 34, 355; 1971) showed that although the micronuclear DNA remains as long pieces, the macronuclear DNA is reduced to small pieces about $0.78 \mu \mathrm{m}$ long. This observation is quite consistent with the observed transection of the chromosomes, and the question arises as to the nature of the reduction and subsequent replication of the macronuclear DNA. Is the replication confined to only a selected portion of the DNA, or are all sequences equally amplified?

This question has now been clearly answered by Bostock and Prescott (Proc. US Nat. Acad. Sci., 69, 139; 1972), who have extracted the micronuclear and macronuclear DNAs and banded them in an analytical $\mathrm{CsCl}$ equilibrium gradient. The micronuclear DNA quite clearly has at least four different density components at $1.699,1.701,1.704$ and $1.709 \mathrm{~g} / \mathrm{ml}$., whereas macronuclear DNA is an almost symmetrical band with a peak at $1.701 \mathrm{~g} / \mathrm{ml}$. Bostock and Prescott exclude the possibility that the extra DNA bands seen in the micronuclear DNA preparations are mitochondrial DNA or DNA from food vacuoles in the Stylonychia cytoplasm, and they are careful to exclude the possibility of polysaccharide contaminants.

It seems therefore that the transformation of the micronucleus to the macronucleus has entailed massive amplification of a selected density species. Whether or not the new macronucleus lacks copies of the other micronuclear DNA density species, as the authors claim, is not quite so clear because their presence would have been obscured by the amplified copies of the $1.701 \mathrm{~g} / \mathrm{ml}$. species. The answer to this question will not be apparent until sufficient DNA can be extracted from the micronucleus for hybridization experiments. The fact remains that Stylonychia presents a very clear example of selective amplification, although the manner in which this occurs remains a mystery.

\title{
Separation of Cytotoxic Cells by Adherence
}

Separation of populations of lymphocytes into two different piles is a useful exercise which is greatly facilitating the study of heterogeneity of lymphoid cell populations and also understanding of the cellular basis of immune responses. One of the first methods, devised by Wigzell, involved the passage of primed or non-primed lymphoid cells down antigen coated columns. The cells which emerged from the bottom of the column were shown to have been depleted of the capacity to respond to the antigen on the column. More recently, A. Basten, J. Sprent and J. F. A. P. Miller (Nature New Biology, 235,$178 ; 1972$ ) have shown that if lymphoid cells are incubated in serum containing antibody and subsequently passed down a column coated with the antigen for which the antibody is specific some lymphocytes are retained in the column. The method of Wigzell removes B cells which have the capacity to respond to a particular antigen ; that of Basten et al. also removes B cells but probably all of them in a given cell population because $B$ cells have the capacity to bind immunoglobulin. In next Wednesday's Nature New Biology (February 23), Clark and Kimura and Lonai, Wekerle and Feldman describe similar methods but ones in which the basis of separation is the capacity to respond to foreign cells in vitro.

If rat lymph node cells are plated out onto mouse fibroblast monolayers in vitro, it can be observed that a proportion of the plated cells becomes adherent to the monolayer within the first day. If the non-adherent cells are removed and plated onto a fresh monolayer of the same phenotype they can be shown to develop less cytotoxic capacity than that demonstrated by the adherent cells. Although the capacity of the supernatant cells to respond to the specific target is reduced, however, these cells are still capable of responding against cells in an antigenically different monolayer. The ability of the adherent cells to respond to the monolayers of the sensitizing cells is amply demonstrated; what is not so clear is whether the adherent cells are restricted in their capacity to respond to a different target. Clark and Kimura's results suggest no such restriction and in fact hint at heightened capacity to respond-this could, however, be attributed to cross-reactions because of some antigens held in common by the different target layers.

The adherence itself commences in as little as 30 minutes after the initiation of the cultures. Various replating experiments are described in which supernatant cells were successively absorbed on monolayers, thus completely eliminating their capacity to respond to the specific target, and reducing their capacity to respond to any target.

The method described by these authors is broadly similar to that recently published by Sprent and Miller (Nature New Biology, 234, 195 ; 1971) in a completely in vivo situation. Sprent and Miller showed that when parental thoracic duct lymphocytes were injected into irradiated $F_{1}$ recipients the cells that emerged in the thoracic duct were restricted in their capacity to respond, being able only to react against antigens similar to those encountered in their first $F_{1}$ recipient. 